\title{
Synchronization in Quotient Network Based on Symmetry
}

\author{
Tao Shaohua ${ }^{*}, 1$, Feng Zhanshen ${ }^{2}$, Luo Jigui ${ }^{2}$ and Zhang Zhili ${ }^{1}$ \\ ${ }^{I}$ School of Information Engineering, Xuchang University, Xuchang, Henan, 461000, P.R. China; ${ }^{2}$ School of Mathematic \\ and Statistics, Xuchang University, Xuchang, Henan, 461000, P.R. China
}

\begin{abstract}
Symmetry is a universal structural property of networks and many real networks have this characteristic. Presently, reducing an original network to a small-size quotient network by means of network symmetry is a promising method for network reduction. This paper studies the synchronization relationship between an original network and its quotient network and concludes that the network and its quotient network reach frequency synchronization simultaneously. We analyze the conclusion in theory and validate it with several simulations on synthetic networks.
\end{abstract}

Keywords: Auto-morphism, orbit, original network, quotient network, symmetry network, synchronization.

\section{INTRODUCTION}

The topologies of real complex networks have been extensively studied [1-11], and many ubiquitous structural characteristics are discovered, such as small-world [1-3], scale-free [4], assortative mixing [7], motif [8] and symmetric structures $[9,10]$. Systematical investigation of the network structural effects on their dynamical processes is of both theoretical and practical importance. One of widely observed phenomena in networked systems is their synchronization behavior, which has received a great deal of attention in the past few decades. Intensive research effort is made towards uncovering the effect of network structure on the network's synchronization behavior, such as the effects of average distance [11,12], heterogeneity [13], clustering [14], and weight distribution. In addition, the relationship of network failure with synchronization [15], generalized synchronization [16], and synchronization performance [16], were also extensively studied.

However, the real networked systems generally have a large size, which makes it difficult to perform theoretical analysis or numerical simulation to explore their synchronization behavior. In a real network, nodes play different roles in the contribution to synchronization behavior of the whole network. Thus, it is desirable if we can find the significant nodes playing important roles for the network synchronization. Network reduction provides us such a way to find these significant skeleton nodes. In a typical network reduction process, we expect to construct a reduced network with smaller size but containing the significant nodes and preserving the important structural properties of the original network. Therefore, as long as a reduced network is constructed with the same synchronization behavior as its original network, the network synchronization behavior can be directly investigated on this reduced network. Computations and analyses on the reduced network will be much easier than on the original network. However, little effort towards this task is known so far.

In this paper, we contribute to constructing a reduced network that can preserve the synchronization behavior of the original network. We find that the quotient network, a reduced network obtained by collapsing the network symmetry structure, can preserve the synchronization behavior of the original network. Besides, when a quotient network achieves synchronization, its original network also achieves synchronization. We demonstrate this by both theoretic analyses and simulation results.

\section{GRAPH SYMMETRY AND QUOTIENT NETWORK}

In this section, we first introduce the preliminary concepts on graph symmetry, then, we illustrate how to obtain the reduced network: quotient network.

\subsection{Graph Symmetry}

A graph or network is denoted by $H=(V, E)$, where $V$ is the set of vertices and $E$ is the set of edges. A one-to-one mapping $a: v \rightarrow v$ is called a permutation on $v$. Obviously, there exists overall $N$ ! (where $N=|V|$ ) permutations on $V$. Among them, some permutations can preserve the adjacency relations for vertex pairs. Such permutations are referred to as automorphism of the graph. More formally, an automorphism of a graph $H$ is a one-to-one mapping $a: v \rightarrow v$ such that $(U, V) \in E$ if and only $(a(u), a(v)) \in E$ let $\operatorname{Aut}(H)$ be the set of all automorphisms of $\mathrm{H}$. For any graph $H$, there exists at least one automorphism: identity automorphism, which maps each vertex to itself. This automorphism is trivial. If a graph has at least one non-trivial automatism, this graph is symmetric. The more automorphisms can be found, the more symmetric the network is.

As an example, we illustrate one symmetric graph in Fig. (1). We can find overall 8 automorphisms in this graph, 
where seven of them are non-trivial. Three non-trivial automorphisms are listed as follows:

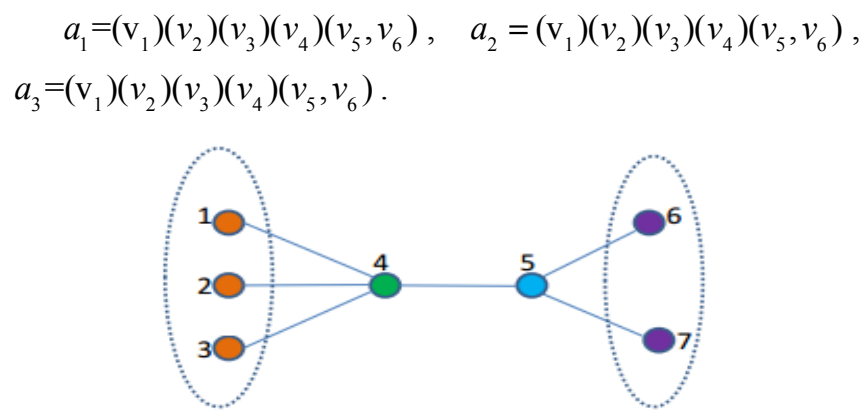

Fig. (1). An example for symmetric network.

Given a graph $H=(V, E)$, for any pair of vertices $u, v \in V$, if there exists an $\alpha \in \operatorname{Aut}(H)$ such that $\alpha(u)=v$, then $u$ is autographically equivalent to $v$. Note that such a relation on the vertex set is an equivalence relation. Thus, we can construct a partition on the vertex set by this equivalence relation. Such a partition is called automorphism partition, each cell of which is called an orbit of $\operatorname{Aut}(H)$. An orbit is trivial if it only contains a single vertex; otherwise, the orbit is non-trivial. In the example shown in Fig. (1), the automorphism partition of the graph is $\pi=\left\{\left\{v_{1}, v_{2}, v_{3}\right\},\left\{v_{4}\right\},\left\{v_{5}\right\},\left\{v_{6}, v_{7}\right\}\right\}$. Vertices in the same orbit are marked with the same color in Fig. (1).

\subsection{Quotient Network}

Actually, the vertices in the same orbit or cell have the same neighborhood structure, and thus they tend to share similar properties, such as degree, eigenvector centrality and clustering coefficient. Therefore, vertices in the same orbit are structurally equivalent to each other. For many real networks with a non-trivial automorphism, those vertices in the same orbit are redundant in the sense that they play the same structural roles. Hence, these vertices are replaceable with each other from the perspective of structure. Thus, structural equivalence implicated by the automorphism partition offers us a convenient way to reduce network size by coarsegraining the vertices within the same orbit. The reduced network derived in this way is referred to as quotient network [17] By only coarse-graining equivalent vertices in the same orbit, all the structural information of the original network are reserved in the quotient network. The quotient network is thus the structural skeleton of its original network, and many characteristics of the original network are preserved in the quotient network.

More formally, let $\ddot{\mathbf{A}}=\left\{\Delta_{1}, \Delta_{2}, \ldots, \Delta_{s}\right\}$ be the automorphism partition of graph $H$. Clearly, the number of neighbors in $\Delta_{j}$ of a vertex $v \in \Delta_{i}$ is $q_{i j}$, which is constant. Then, the quotient network $Q$ of $H$ under the action of $\operatorname{Aut}(H)$ essentially is an edge-weighted multi-digraph (i.e., there may exist multiple edges among a pair of vertices and each edge is weighted and directed) with orbits of $H$ as the vertex set and adjacency matrix consisting of $q_{i j}$. It was already shown that many real networks can be reduced to non-trivial quotient networks, each of which is significantly smaller than the size of the original network but preserves the most important structural characteristics of the original network [17]. In this sense, the quotient network is a good reduction of the original network.

A graph's quotient network can be easily constructed if the automorphism partition of this graph is already given. In this paper, NAUTY [17] tools are adopted to obtain the automirphism partition of a graph, and thus we just need to coarse-grain each orbit of the graph into a single vertex so as to construct the quotient network. Take the graph shown in Fig. (1) for example, we give out its quotient network in Fig. (2). The quotient network is obtained by coarse-graining orbits as follows:

$$
\left\{v_{1}, v_{2}, v_{3}\right\} \rightarrow v_{1},\left\{v_{4}\right\} \rightarrow v_{2},\left\{v_{5}\right\} \rightarrow v_{3},\left\{v_{6}, v_{7}\right\} \rightarrow v_{4},
$$

Where the left parts are orbits in the original graph and the right parts are vertices in the quotient network. Note that each edge is directed and weighted in the quotient network, which represents the number of the neighbors for a vertex in one orbit directed to another orbit.

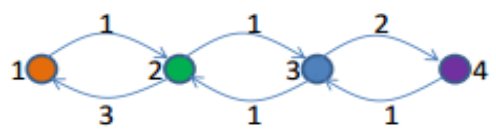

Fig. (2). Quotient network.

\section{SYNCHRONIZATION OF NETWORK}

Kuramoto model [18] is described by a simple model of $N$ mutually coupled oscillators having different intrinsic frequencies. For a graph $G(V, E)$ with $|V|=N$ vertices, we can define a connectivity matrix $A=\left[a_{i j}\right](1 \leq i, j \leq N)$ such that $a_{i j}=1$ if vertices $i, j$ are connected and $a_{i j}=0$ otherwise. Let $\phi_{i}$ be the phase of vertex $i, \phi_{i} \in[0,2 \pi)$ and $\frac{d \varphi_{i}}{d t}$ be the $i-t$ th phase rate of change. Then, the Kuramoto model for graph $G$ can be formally given by the dynamic equation of $\frac{d \varphi_{i}}{d t}$ as

$\frac{d \varphi_{i}}{d t}=\omega_{i}-\frac{\sigma}{N} \sum_{j} a_{i j} \sin \left(\varphi_{i}-\varphi_{j}\right), i=1,2 \ldots N$

where, $\omega_{i}$ is the intrinsic frequency of vertex $i$ and $\sigma$ is the coupling strength of connected oscillators. In general, $\omega_{i} \in[-0.5,0.5]$ is chosen from the Gaussian distribution with unit variance.

The order parameter $M$ is usually used to evaluate the macroscopic synchronization. $M$ is usually defined as 
$M=\left|\frac{1}{N} \sum_{1 \leq j \leq N} e^{i \phi_{i}}\right|$

If all of the oscillators are in the same phases, then $M=$ 1 ; otherwise, $M=0$. When $M_{\text {limit }_{t \rightarrow \infty}}=\rho$, the network approaches frequency synchronization, where, $\rho$ is a positive constant. In the following, unless clearly specified, network synchronization refers to the frequency synchronization.

\section{SYNCHRONIZABLITY ANALYSIS}

It is very difficult to measure the synchronizablity of Eq. (1) either in theoretic analysis or numerical simulation, because synchronizablity has higher dimensions. It is also very difficult to analyze the synchronization when the network size is very large. Hence, it is necessary to decompose Eq. (1) and make its dimension lower so that the judgment of system synchronization would be much easier. To measure the synchronized state, we first need to rewrite Eq. (1) with some new parameters. Let $\phi=\omega_{t}+\phi_{0}, \varepsilon=\phi_{i}-\phi$ be the differences of the phases and the average phase, respectively. Let $\phi=\frac{1}{N} \sum_{1 \leq j \leq N} \phi_{j}, \omega=\frac{1}{N} \sum_{1 \leq j \leq N} \omega_{j}, \varepsilon_{i}=\phi_{i}-\omega_{t}$, here $\phi$ is the average phase and $\omega$ is the average frequency rate. Now, Eq. (1) can be rewritten as systematic error expression, shown in Eq. (3). Zero systematic error is equivalent to the synchronization of both the original network and the quotient network in the sense that they reach synchronization simultaneously.

$\varepsilon=\omega_{i}-\omega-\frac{\sigma}{N} \sum_{1 \leq j \leq N} a_{i j} \sin \left(\varepsilon_{i}-\varepsilon_{j}\right)$

when, $t \rightarrow \infty, \omega_{i}-\omega-\frac{\sigma}{N} \sum_{1 \leq j \leq N} a_{i j} \sin \left(\varepsilon_{i}-\varepsilon_{j}\right)=0$ and Eq.(3) has solution when the right side of Eq.(3) reaches an equilibrium state. Eq. (1) approaches frequency synchronization state when Eq. (3) approaches partial stability state on the equilibrium point. Here, the concepts of partial stability and also partial synchronization are referred to as the definitions in [19].

Theorem 1 If Eq. (3) satisfies that

(1) The linearized matrix of Eq. (3) has solution on equilibrium point $\left(\varepsilon_{1}^{*}, \varepsilon_{2}^{*}, \ldots \varepsilon_{N}^{*}\right)$;

(2) $\left|\varepsilon_{i^{*}}-\varepsilon_{j^{*}}\right|<\frac{\pi}{2}$;
Eq. (1) reaches partial frequency synchronization if Eq. (3) is partial stable on equilibrium point $\left(\varepsilon_{1}^{*}, \varepsilon_{2}^{*}, \ldots \varepsilon_{N}^{*}\right)$ where the linearized matrix on the equilibrium point is as follows:

$\Lambda=\left[\begin{array}{cccc}-\sum_{1 \leq j \leq N} \Delta \varepsilon_{1 j}^{*} & \Delta \varepsilon_{12}^{*} & \ldots & \Delta \varepsilon_{1 N}^{*} \\ \Delta \varepsilon_{21}^{*} & -\sum_{1 \leq j \leq N} \Delta \varepsilon_{2 j}^{*} & \ldots & \Delta \varepsilon_{2 N}^{*} \\ . . & . . & . . & . . \\ \Delta \varepsilon_{N 1}^{*} & \Delta \varepsilon_{N 2}^{*} & \ldots & -\sum_{1 \leq j \leq N} \Delta \varepsilon_{N j}^{*}\end{array}\right]$

Here,

$\Delta \varepsilon_{1 j}^{*}=a_{1 j} * \cos \left(\varepsilon_{1}^{*}-\varepsilon_{j}^{*}\right), \Delta \varepsilon_{12}^{*}=a_{12} * \cos \left(\varepsilon_{1}^{*}-\varepsilon_{2}^{*}\right), \ldots \Delta \varepsilon_{N j}^{*}=a_{N j}$ $* \cos \left(\varepsilon_{N}^{*}-\varepsilon_{j}^{*}\right)$ If in a connected network and under the condition $\left|\varepsilon_{i}^{*}-\varepsilon_{j}^{*}\right|<\frac{\pi}{2}$, we can get the real parts of all eigenvalues $\lambda_{i} \leq 0$ of matrix $\Lambda$, and it at most has one zero root, then the dynamic network (3) approaches partial stability on the equilibrium point [20]. This means that when the dynamic network reaches partial stability on the equilibrium point, it is equivalent to the original system's synchronism. We call this synchronization the generalized phase synchronism or the generalized frequency synchronization.

Given the adjacent matrix $T=\left[t_{i j}\right]$ of an undirected network, its coupling matrix $A=\left[a_{i j}\right] \in R^{n \times n}$ is defined

$$
\text { as } a_{i j}= \begin{cases}-\sum_{1 \leq j \leq N} t_{i j} & \text { if } i=j, \\ 1 & \text { if } i \text { and } j \text { are connected, } \\ 0 & \text { otherwise }\end{cases}
$$

Given the adjacent matrix $T=\left[t_{i j}\right]$ of an undirected network, the coupling matrix of its quotient network can be defined as

$$
b_{i j}=\left\{\begin{array}{cc}
-\sum_{1 \leq j \leq N} b_{N j} & \text { if } i=j \\
1 & \text { if } i \text { and } j \text { connected }, \\
k_{i} & \text { if } r \text { and } j \text { connected, and } r=i, \\
0 & \text { otherwise }
\end{array}\right.
$$

Where, $k_{i}$ is the node number of the $i$-th orbit in the original network and the $i$-th orbit is presented by the node $r$ in the quotient network.

In a complex network, each node is considered to be oscillator in the Kuramoto model. In the original network, if an orbit only contains a single oscillator, the orbit is trivial; otherwise, it is non-trivial. If $\phi_{\mathrm{i}}$ is the $i$-th trivial phase rate of change, usually its nonlinear equation is 
$\varphi_{i}=\omega_{i}-\frac{\sigma}{N} \sum_{1 \leq j \leq N} a_{i j} \sin \left(\varphi_{i}-\varphi_{j}\right)$

In a non-trivial orbit which contains more than one oscillator, each oscillator interacts with all the others. The oscillator's nonlinear equations in the same orbit are

$$
\begin{gathered}
\varphi_{j}=\omega_{j}-\frac{\sigma}{N} a_{j 1} \sin \left(\varphi_{j}-\varphi_{1}\right) \\
\varphi_{j}=\omega_{j}-\frac{\sigma}{N} a_{j 2} \sin \left(\varphi_{j}-\varphi_{2}\right) \\
\varphi_{j}=\omega_{j}-\frac{\sigma}{N} a_{j 3} \sin \left(\varphi_{j}-\varphi_{3}\right) \\
\vdots \\
\varphi_{j}=\omega_{j}-\frac{\sigma}{N} a_{j r} \sin \left(\varphi_{j}-\varphi_{r}\right)
\end{gathered}
$$

Let $j$ be the common coupling vertex of the nodes $1,2,3, \ldots r$. Since the nodes $1,2,3, \ldots r$ in the same orbit have equal state, we have $\varepsilon_{i}=\varepsilon_{j}$ in Eq. (3). The nodes $1,2,3, \ldots r$ in the same orbit can be replaced by $r$, then, we can reformulate the above equation as;

$\varphi_{j}=\omega_{j}-\frac{\sigma}{N} a_{j r} k_{i} r \sin \left(\varphi_{j}-\varphi_{r}\right)$

Based on the stability analysis on the equilibrium point and the Theorem 1, we conclude that when the reduced network approaches the synchronized state, the original network also approaches synchronization under the given conditions.

\section{ILLUSTRATION EXAMPLE}

We use the network shown in Fig. (1) as an example to illustrate our theoretic results. The network coupling matrix is:

$$
A=\left[\begin{array}{ccccccc}
-1 & 0 & 0 & 1 & 0 & 0 & 0 \\
0 & -1 & 0 & 1 & 0 & 0 & 0 \\
0 & 0 & -1 & 1 & 0 & 0 & 0 \\
1 & 1 & 1 & -4 & 1 & 0 & 0 \\
0 & 0 & 0 & 1 & -3 & 1 & 1 \\
0 & 0 & 0 & 0 & 1 & -1 & 0 \\
0 & 0 & 0 & 0 & 1 & 0 & -1
\end{array}\right]
$$

The dynamic equations are

$$
\begin{gathered}
\varphi_{1}=\omega_{1}-\frac{\sigma}{N} a_{41} \sin \left(\varphi_{4}-\varphi_{1}\right) \varphi_{2}=\omega_{2}-\frac{\sigma}{N} a_{42} \sin \left(\varphi_{4}-\varphi_{2}\right) \\
\varphi_{3}=\omega_{3}-\frac{\sigma}{N} a_{43} \sin \left(\varphi_{4}-\varphi_{3}\right) \varphi_{4}=\omega_{4}-\frac{\sigma}{N} a_{54} \sin \left(\varphi_{5}-\varphi_{4}\right)
\end{gathered}
$$

$$
\begin{aligned}
\varphi_{5} & =\omega_{5}-\frac{\sigma}{N} a_{65} \sin \left(\varphi_{6}-\varphi_{5}\right) \varphi_{6}=\omega_{6}-\frac{\sigma}{N} a_{56} \sin \left(\varphi_{5}-\varphi_{6}\right) \\
\varphi_{7} & =\omega_{7}-\frac{\sigma}{N} a_{57} \sin \left(\varphi_{5}-\varphi_{7}\right)
\end{aligned}
$$

For the linearized dynamic equation, the coupling matrix $A$ has the solution $\varepsilon_{1}^{*}=-0.725, \varepsilon_{2}^{*}=-0.1172, \varepsilon_{3}^{*}=-0.4259$, $\varepsilon_{4}^{*}=-0.2569, \quad \varepsilon_{5}^{*}=-0.6597, \quad \varepsilon_{6}^{*}=-0.3570, \quad \varepsilon_{7}^{*}=-0.7785$ on the equilibrium point $\left(\varepsilon_{1} \varepsilon_{2} \varepsilon_{3} \varepsilon_{4} \varepsilon_{5} \varepsilon_{6} \varepsilon_{7}\right)$. Moreover, $\left|\varepsilon_{i}^{*}-\varepsilon_{j}^{*}\right|$ are $0.1690,0.2338,0.0689,0.3525,0.1396,0.1690$, and 0.0 , respectively, which satisfy $\left|\varepsilon_{i}^{*}-\varepsilon_{j}^{*}\right|<\frac{\pi}{2}$ with only one zero root. The dynamic equation of the original network has the solutions and $\left|\varepsilon_{i}^{*}-\varepsilon_{j}^{*}\right|<\frac{\pi}{2}$ satisfying Theorem 1, which means that the dynamic equation of the original network is partial stable on the equilibrium point $\left(\varepsilon_{1}^{*}, \varepsilon_{2}^{*}, \varepsilon_{3}^{*}, \varepsilon_{4}^{*}, \varepsilon_{5}^{*}, \varepsilon_{6}^{*}, \varepsilon_{7}^{*}\right)$.

We now reduce the original network to its quotient network. Notice that nodes 1, 2, and 3 are in the same orbit, and nodes 6 and 7 are in the same orbit. Hence, nodes 1, 2, and 3 can be replaced by node 1 , and node 4 with $2 ; 5$ with 3 ; and 6 , and 7 with 4 . Weighted and directed network topology of the quotient network is shown as Fig. (2). The coupling matrix $B$ of the quotient network are

$$
B=\left[\begin{array}{cccc}
-1 & 1 & 0 & 0 \\
3 & -4 & 1 & 0 \\
0 & 1 & -3 & 2 \\
0 & 0 & 1 & -1
\end{array}\right]
$$

The dynamic equations of the quotient network are

$$
\begin{gathered}
\varphi_{1}=\omega_{1}-\frac{\sigma}{N} a_{21} \sin \left(\varphi_{2}-\varphi_{1}\right) \\
\varphi_{2}=\omega_{2}-3 \frac{\sigma}{N} a_{12} \sin \left(\varphi_{1}-\varphi_{2}\right) \varphi_{3}=\omega_{3}-2 \frac{\sigma}{N} a_{43} \sin \left(\varphi_{4}-\varphi_{3}\right) \\
\varphi_{4}=\omega_{4}-\frac{\sigma}{N} a_{34} \sin \left(\varphi_{3}-\varphi_{4}\right)
\end{gathered}
$$

For the linearized dynamic equation of the quotient network on the equilibrium point $\left(\varepsilon_{1}, \varepsilon_{2}, \varepsilon_{3}, \varepsilon_{4}\right)$, the coupling matrix $B$ has the solution $\varepsilon_{1}^{*}=-0.2757, \varepsilon_{2}^{*}=-0.1377$, $\varepsilon_{3}^{*}=-0.6042, \quad \varepsilon_{4}^{*}=-0.6044$ and $\left|\varepsilon_{i}^{*}-\varepsilon_{j}^{*}\right|$ are 0.1380 , $0.3285,0.3286$, and 0.0 , respectively. The equation satisfies $\left|\varepsilon_{i}^{*}-\varepsilon_{j}^{*}\right|<\frac{\pi}{2}$ and has at most one zero roots. The dynamic equation of the quotient network has the solutions and $\left|\varepsilon_{i}^{*}-\varepsilon_{j}^{*}\right|<\frac{\pi}{2}$ which means the dynamic equation of the quotient network achieves partial stability on the equilibrium 
point $\left(\varepsilon_{1}^{*}, \varepsilon_{2}^{*}, \varepsilon_{3}^{*}, \varepsilon_{4}^{*}\right)$ and approaches the frequency synchronization state.

Fig. (3) shows the results with coupling strength $\sigma=0.5$.

We can see that $\sigma=0.5$ is the smallest coupling strength with which both networks reach the frequency synchronization at the same time. From a practical viewpoint, we analyzed these parameters $\sigma$ and $\omega$ as follows:

When phase $\phi_{i}$ is fixed, we examine the critical point of the coupling strength $\sigma_{c}$. Here, $\sigma_{c}=0.5$ is a critical point at which the original network and the quotient network reach the frequency synchronization simultaneously. When the coupling strength satisfies $0.3 \leq \sigma<0.5$, the original network can reach synchronization, but the quotient network cannot, as shown in Fig. (4).

Neither of the networks can reach frequency synchronization when $\sigma<0.3$. In general, for connected networks, if 


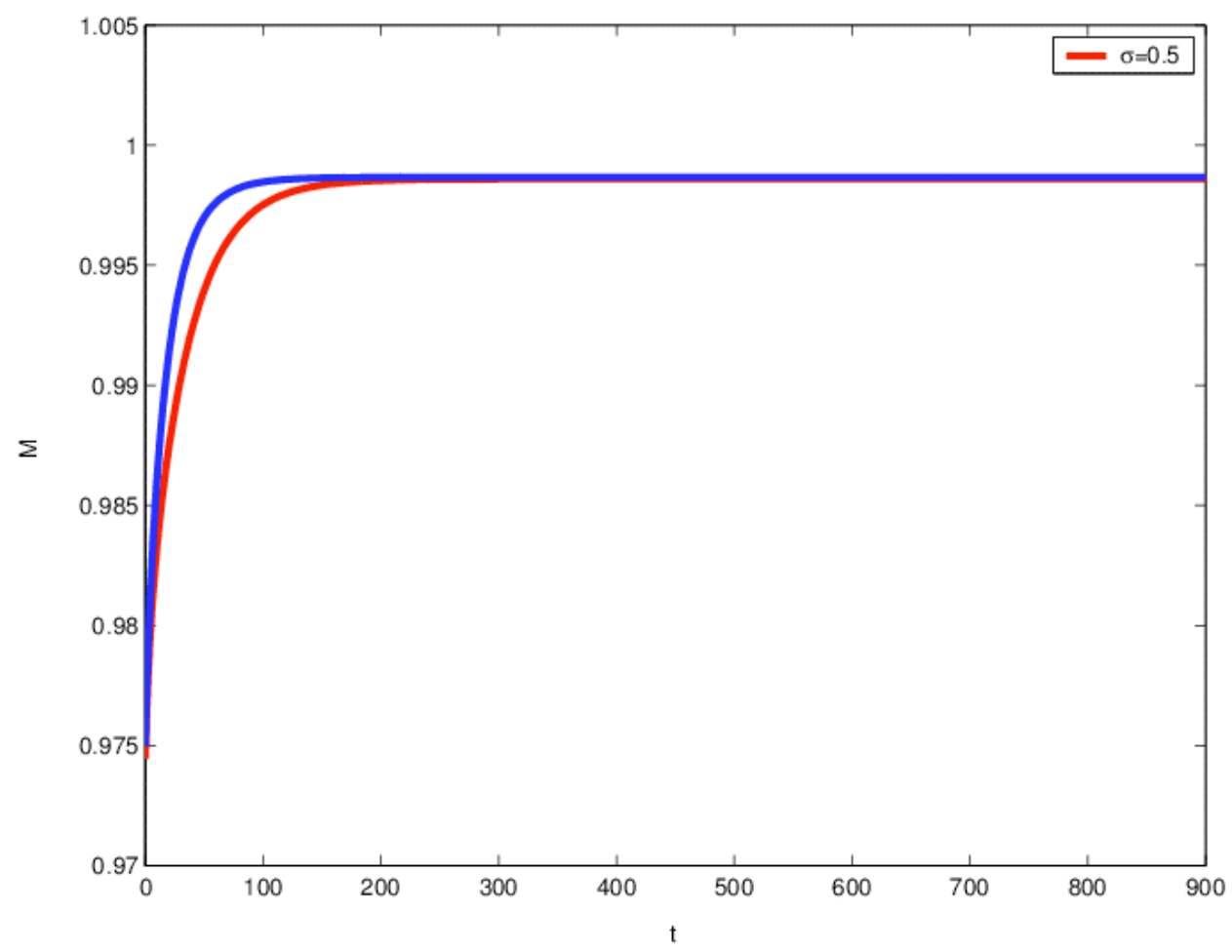

Fig. (3). The coupled strength $\sigma$ vs. time, the coupled strength $\sigma=0.5$. The blue line represents the original network and the red line represents the quotient network.

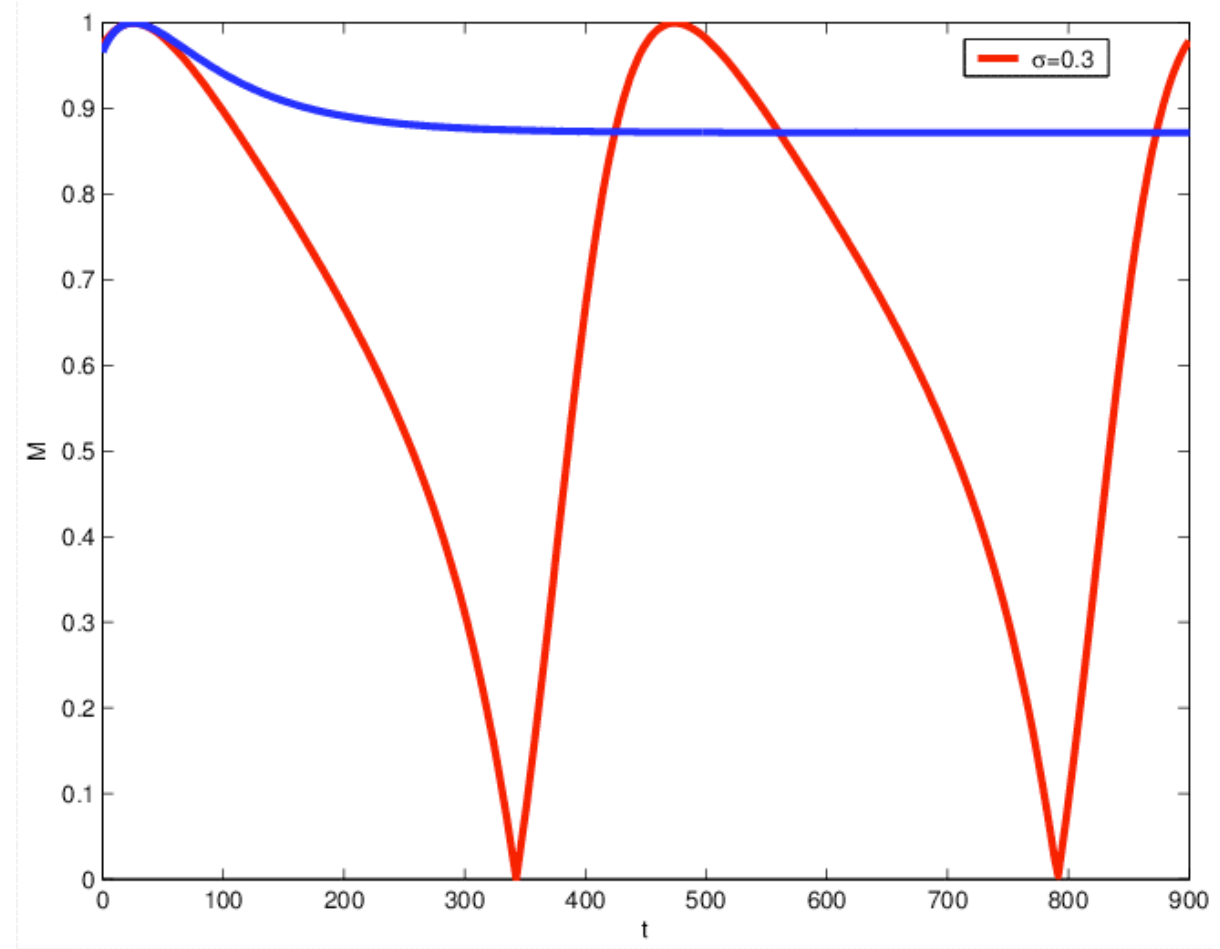

Fig. (4). The original network and the quotient network cannot reach synchronization simultaneously. The blue line represents the original network and the red line represents the quotient network.

the difference $\omega_{i}-\omega$ is small and the $\delta$ value is moderate, then the order parameter $M$ tends to be stable at a positive constant where the network achieves synchronization. If the coupling strength $\sigma$ is too big and the order parameter $M$ is a positive constant, both of the two networks can reach frequency synchronization, however, this is of no practical significance. Fig. (5) shows the synchronized states of the original network and the quotient network. ON represents 


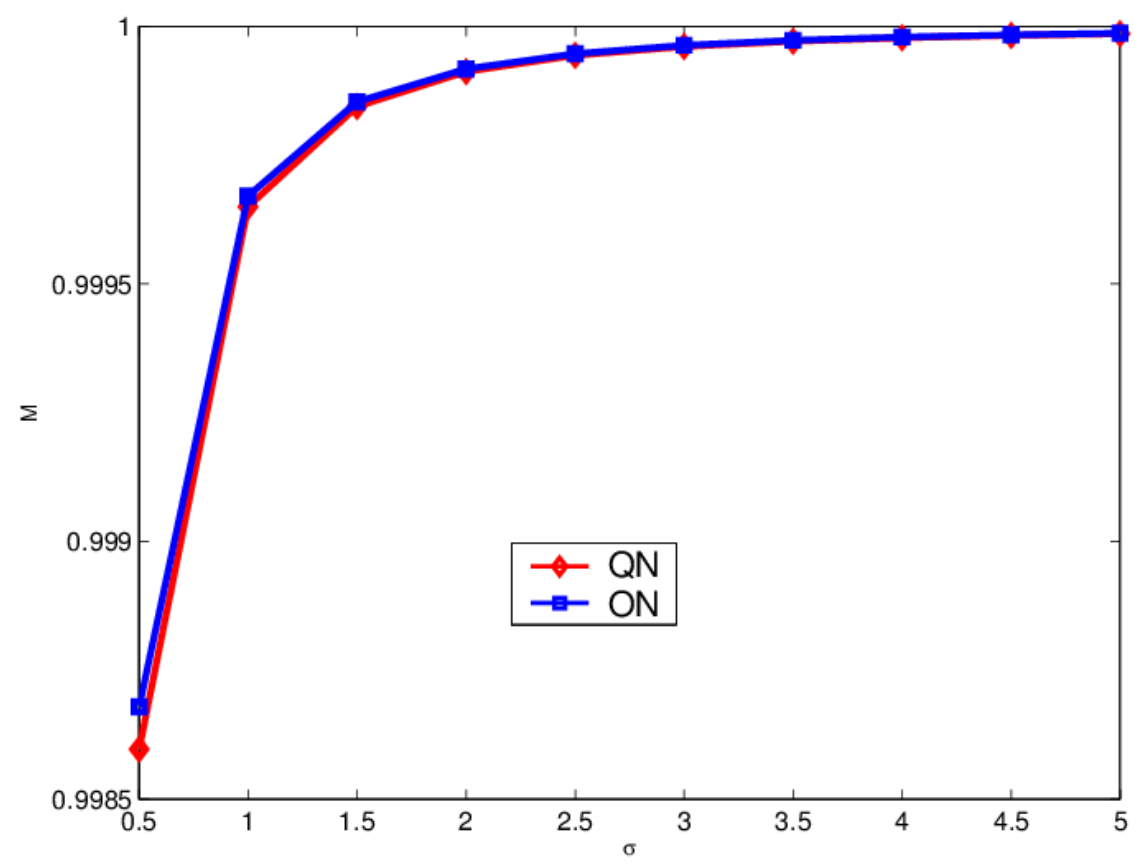

Fig. (5). Order parameter $M$ and coupled strength $\sigma$. The blue line is the original network and the red line is the quotient network.

the original network and QN represents the quotient network. From this numerical simulation, we clearly see that the synchronizations of the original network and the quotient network have slight phase differences at the early stage. This is because there exist a fixed phase difference between the two equilibrium points.

All oscillators in both the original and the quotient networks keep the same pace gradually, and both of the two networks achieve the same synchronization with the coupling strength $\sigma>\sigma_{c}=0.5$. Hence, the original network and the quotient network can reach the same frequency synchronization. On the other hand, when both of the two networks approach synchronization, the graph shows that the order parameter $M$ of the original network is bigger than the quotient network. Hence, from the perspective of phase synchronization, the synchronization of the original network is slightly better than that of the quotient network when the coupling strength of the former is smaller than that of the latter. Generally speaking, the synchronization would be better if the order parameter $M$ is bigger and the phase difference is smaller. The phase synchronization is a kind of special frequency synchronizations, and the conditions to reach synchronization are relatively high.

\section{CONCLUSION}

Many real networks contain abundant structural equivalence, which enables us to derive the reduce network, quotient network, by collapsing the structural equivalent nodes in a symmetric network. In this paper, we conclude that a network and its quotient network reach frequency synchronization simultaneously for most real complex networks. We analyze this conclusion in theory and validate it with simulations on synthetic networks. The conclusion implies that we can get the synchronization behavior of an original network just from its quotient network. Since quotient network size is usually smaller than its original network, it is very easy to analyze the synchronization and carry out other measurements on it. The numerical simulations show that when the quotient network reaches synchronization, the original network also approaches synchronization. The conclusion of this paper will help to reduce the cost for studying a network's synchronization behavior because the quotient networks have smaller sizes without sacrificing the structural information of their original networks.

\section{CONFLICT OF INTEREST}

The authors confirm that this article content has no conflict of interest.

\section{ACKNOWLEDGEMENTS}

This work was supported by the Foundation Name under Grant No. (122102210544 and No.12A510022 and No. (132300410349)) and natural foundation fund (No. U1304403).

\section{REFERENCES}

[1] A. Nematzadeh, E. Ferrara, A. Flammini and Y.-Y. Ahn. " Optimal network modularity for information diffusion", PRL. vol. 113, pp. 088701:23-088701:33, August 2014.

[2] L.Weng, F.Menczer, and Y.-Y.Ahn. "Information diffusion on online social networks", Sci. Rep., vol. 3, pp. 345-356, August 2013.

[3] D.J. Watts and S.H. Strogatz. "Collective dynamics of 'smallworld' networks", Nature, vol. 393, pp. 440-442, February 1998.

[4] A.-L.Barab_asi and R.Albert "Emergence of scaling in random networks", Science, vol. 86, pp. 509-512, Setpember 1999.

[5] Y.C. Zhang, M.A. A-Alaoui and C. Bertelle, "Local Nash Equilibrium in Social networks", Sci. Rep., vol. 4, pp. 6224-6228, August 2014.

[6] M.E.J. Newman, "Mixing patterns in networks", Phys. Rev. E, vol. 67, pp. 026126:348-026126:354, February 2003.

[7] M.E.J. Newman, " Detecting community structure in networks", Eur. Phys. J. B, vol. 38, pp. 321-330, May 2004.

[8] B. Zheng, B. Wu, and D. Li, "S simple model clarifies the complicated relationships of complex networks", Sci. Rep., vol. 4, pp. 6197-5106, August 2014. 
[9] B.D. MacArthur, R.J.S. anchez-Garc, and J.W. Anderson, "Symmetry in complex networks", Discr Appl Math, vol. 156, pp. 35253531 , July 2008

[10] Y.H. Xiao, M.M. Xiong, W. Wang, and H. Wang, "Emergence of symmetry in complex networks", Phys. Rev. E, vol. 77, pp. 066108:1-066108:10, September 2008.

[11] T. Martin, X. Zhang, and M.E.J. Newman, " Localization and centrality in networks", Phys. Rev. E, vol. 90, no. 5, pp. 052808, 2014.

[12] H.D. Hasegawa, "Dynamical mean-field approzimation to smallworld networks of spiking neurons: From local to global and/or from regular to random couplings", Phys. Rev. E, vol. 70, pp. 066107, December 2004.

[13] A.E. Motter, C.S. Zhou, and J. Kurths, "Enhanceing complexnetwork synchronization " Euro. Phys. Lett., vol. 69, pp. 334-338, June 2005 .

[14] Y. Kim, Y.J. Ko, and S.-H. Yook, "Structural properties of the synchronized cluster on complex networks", Phys. Rev. E Stat. Nonlin. Soft Matter Phys., vol. 81, pp. 011139, February 2010.
[15] Q.Y. Wang, Z.S. Duan, G.R. Chen, and Z.S. Feng, " Synchronization transition in Gap-Junction-Coupled leech neurons ", Physica $A$, vol. 387, pp. 4404-4410, May 2008

[16] W. Yu, G. Chen, J. Lü, and J. Kurths, "Synchronization via pinning control on general complex networks", SIAM J. Control Optim., vol. 51, pp. 1395-1416, June 2013

[17] Y.H. Xiao, B.D. MacArthur, H. Wang, and W. Wang," Network quotients: structural skeletons of complex systems", Phys. Rev. E, vol. 78, pp. 046102, March, 2008

[18] Z.Wu and X. Fu, " Combination synchronization of three different order nonlinear systems using active backstepping design", Nonlinear Dynam., vol. 73, pp. 1863-1872, February 2013.

[19] L.L. Bonilla J.A. Acebron, C.J.P. Vicente, F. Ritort, and R. Spigler, "The Kuramoto model: a simple paradigm for synchronization phenomena," Rev. Mod. Phys, vol. 77, pp. 137-185, July 2005.

[20] J.H. Lü, X.G.Yu, and G.R.Chen, "Chaos synchronization of general complex dynamical networks", Phys. A, vol. 302, pp. 334-281, August 2004.

Received: November 24, 2014

Revised: December 17,2014

Accepted: December 19,2014

(C) Shaohua et al.; Licensee Bentham Open.

This is an open access article licensed under the terms of the Creative Commons Attribution Non-Commercial License (http://creativecommons.org/licenses/by-nc/3.0/) which permits unrestricted, non-commercial use, distribution and reproduction in any medium, provided the work is properly cited. 